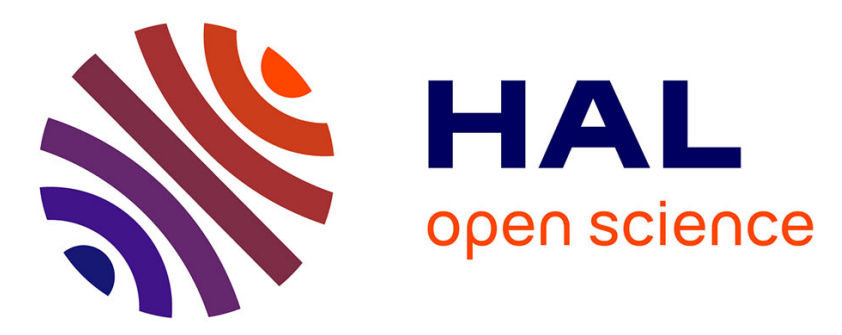

\title{
Experimental Study of the Precessing Vortex Core Impact on the Liquid Fuel Spray in a Gas Turbine Model Combustor
}

Antoine Renaud, Sebastien Ducruix, Laurent Zimmer

\section{To cite this version:}

Antoine Renaud, Sebastien Ducruix, Laurent Zimmer. Experimental Study of the Precessing Vortex Core Impact on the Liquid Fuel Spray in a Gas Turbine Model Combustor. Journal of Engineering for Gas Turbines and Power, 2019, 10.1115/gt2019-91619 . hal-02312909

\section{HAL Id: hal-02312909 \\ https://hal.science/hal-02312909}

Submitted on 11 Oct 2019

HAL is a multi-disciplinary open access archive for the deposit and dissemination of scientific research documents, whether they are published or not. The documents may come from teaching and research institutions in France or abroad, or from public or private research centers.
L'archive ouverte pluridisciplinaire HAL, est destinée au dépôt et à la diffusion de documents scientifiques de niveau recherche, publiés ou non, émanant des établissements d'enseignement et de recherche français ou étrangers, des laboratoires publics ou privés. 


\title{
EXPERIMENTAL STUDY OF THE PRECESSING VORTEX CORE IMPACT ON THE LIQUID FUEL SPRAY IN A GAS TURBINE MODEL COMBUSTOR
}

\author{
Antoine Renaud; Sébastien Ducruix, Laurent Zimmer \\ Laboratoire EM2C \\ CNRS, CentraleSupelec, Université Paris-Saclay \\ 91190 Gif-sur-Yvette \\ France \\ Email: antoine.renaud@centralesupelec.fr
}

\begin{abstract}
Despite being good candidates for the reduction of pollutant emissions from gas turbines, burners operating in Lean Premixed Prevaporized regimes often face stability issues and can be sensitive to perturbations. The swirling flow used to aerodynamically stabilize the flame can also lead to the appearance of a large-scale coherent flow structure known as the Precessing Vortex Core (PVC). In the present study, a swirl-stabilized combustor fed with liquid dodecane is studied at a globally lean operating condition with the help of high-speed diagnostics and Dynamic Mode Decomposition (DMD) as the main post-processing method. It is shown that the trace of a PVC originating inside the injector is still present in the fuel spray at the entrance of the chamber even though the aerodynamical structure itself is not detectable anymore. The perturbation of the fuel spray is then transmitted to the flame through local equivalence ratio fluctuations. It is observed that the PVC trace on the spray and thus on the flame can be suppressed by air flow modulations generated by a siren device. The suppression of this trace is shown to come from a decay of the aerodynamical structure itself rather than by a change in fuel mixing or vaporization. Analysis of the characteristic frequency of the PVC shows a frequency spread indicating a loss of coherence of the structure with the high amplitude air flow rate fluctuations.
\end{abstract}

\section{INTRODUCTION}

Because of raised awareness about pollutant emissions and the necessity to reduce them, increasingly restrictive emission norms have been set up for aircraft engines in recent years. Manufacturers now need to move away from the traditionally used diffusion flames in order to meet the goals set by the regulation [1]. A candidate for this purpose is the switch to Lean Premixed Prevaporized (LPP) regimes which have a strong potential in terms of carbon monoxide $(\mathrm{CO})$ and nitrogen oxide (NOx) emission reduction [2-4]. It consists in providing a uniform lean mixture of air and fuel that would burn with lower temperatures, thus reducing the thermal-NOx emissions. A key feature of lean premixed combustion is however its sensitivity to external perturbations leading to flame stabilisation issues [5-7] or to the triggering of thermo-acoustic instabilities $[8,9]$.

In aeroengines, flame stabilisation is aerodynamically performed with the help of swirling flows. They usually create a vortex breakdown, generating recirculation zones and thus providing low velocity regions and hot burnt gases aiming at permanently reigniting the flame. Since lean premixed flames are sensitive to perturbations, large scale coherent flow structures may disturb them. Strongly swirling flows often exhibit a helical structure known as a Precessing Vortex Core (PVC) [10-14] which can disturb the combustion process. The occurrence and strength of the PVC depends on numerous parameters: swirl number [15, 16], fuel injection mode or equivalence ratio.

${ }^{*}$ Corresponding author. 
The relationship between PVC and combustion is complex. Most of the time, it is seen that the PVC is damped in reacting conditions $[17,18]$ because of inhomogeneities in density created by the flame $[19,20]$. It is however sometimes observed that the PVC can be strengthened by combustion, depending on the operating conditions [21]. Studies have shown that the PVC acts on the flame and can help in stabilizing it by creating a good mixing between the reactants and the hot burnt gases [22-24] or by acting on the stagnation point of the flow [25]. The existence or absence of a PVC is also often linked with the attached or detached states of flames in a given burner [26-28].

By acting on the flame, the PVC can create local heat release fluctuations [29] which raises the question of its coupling with thermoacoustic instabilities [30-32]. Studies carrying out artificial flow forcing can shed light on the potential interactions between the PVC and global flow field oscillations. When the forcing frequency remains low compared to that of the PVC, several studies showed that the PVC can be damped and even completely destroyed by axial forcing [33-36]. On the other hand, Iudiciani et al. showed that higher frequency forcing (at twice the PVC frequency) can reinforce the PVC [37].

Most of the literature concerning the interaction between PVC and combustion concerns premixed cases with gaseous reactants. In the present study, the burner is operated using a liquid fuel injection. This adds further complexity to the interplay between the fuel, the flame and the flow by introducing atomization, droplets motion and vaporization in the picture. Indeed, previous studies carried out in this burner $[26,38]$ have shown that the flame strongly fluctuates at the PVC frequency when it is attached to the injector in contradiction with numerous literature results indicating that this mode of flame stabilization should damp the PVC [39,40]. Other observations of the present setup [32] have shown that the PVC effect seems reduced when thermo-acoustic instabilities occur.

Further investigations aimed at understanding the previous observations are carried out in the present work. With the help of laser based measurements and powerful post-processing tools such as Dynamic Mode Decomposition (DMD), the interplay between the PVC, the fuel spray and the flame is highlighted. After presenting the experimental setup and the post-processing methods, focus is put in on the characterization of the PVC impact on the flame which is shown to occur through fluctuations of the fuel spray. Finally, the response of the burner and the PVC to external perturbations is studied.

\section{DESCRIPTION OF THE EXPERIMENTS Experimental Setup}

In the BIMER experimental device [26,38], a simplified version of an industrial staged injector is placed in a plenum attached to a $500 \mathrm{~mm}$-long combustion chamber with a square section $\left(150 \times 150 \mathrm{~mm}^{2}\right)$. Tests are performed at atmospheric pressure. The chamber is water-cooled and equipped with silica windows for optical access. The burner is fueled with liquid dodecane, chosen as a kerosene surrogate, and air is preheated (Sylvania SureHeat Max electric preheater) to enhance fuel vaporisation. A cut of the injector is shown in Fig. 1. Liquid fuel is injected through a central pressurized nozzle (MMP 6390-0-0-03, flow number: $1.41 . \mathrm{h}^{-1} . \mathrm{bar}^{-0.5}$ ) that creates

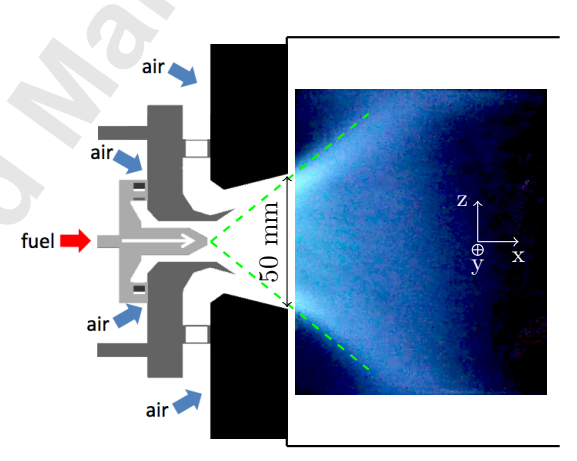

FIGURE 1. Vertical cut of the injection device placed at the entrance of the combustion chamber. Green dashed lines represent the general shape of the hollow-cone spray. A photography (at scale) of the flame is added to show its aspect in the present study.

a hollow cone of droplets. Visualizations of the spray in the chamber as well as inside the injector (see Diagnostics section) show no evidence of fuel filming onto the walls of the injector. The air flow is divided between two stages by the geometrical design of the injector. The upstream stage (light gray in Fig. 1) allows 13.5\% of the air to flow through and is called the pilot stage. It contains a 
swirler with a geometrical swirl number around 0.6. The downstream stage (dark gray) is called the take-off stage. The remaining $86.5 \%$ of the air flow through its swirler with a geometrical swirl number close to 1.

To study the effect of artificial oscillations on the burner, a siren device (CBOne, Graz, Austria) is installed upstream of the plenum. It enables to perform modulations with independently controlled frequency and amplitude [41]. The principle of this siren is to rotate a toothed wheel ( $9 \mathrm{~mm}$-high teeth) in front of a $12 \mathrm{~mm}$-diameter hole through which the air flows. The rotational speed of the wheel controls the frequency of the modulations while their amplitude depends on the relative position between the teeth of the wheel and the hole. This is controlled by an eccentric gear that allows to modify the position of the wheel axis. Since the siren is placed after the preheater, its shaft and bearings are cooled with a flow of air at ambient temperature.

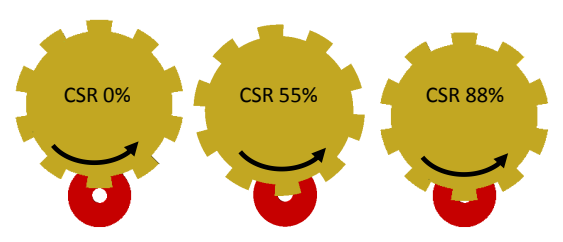

FIGURE 2. Relative positions of the hole and the toothed wheel for different CSR.

To monitor the amplitude of the air flow rate modulations, the Covered Surface Ratio (CSR) is derived from geometrical considerations. It is defined as the ratio between the maximum area of the hole covered by the tooth to the area of the hole when it is not covered by a tooth. For example, CSR 55\% means that 55\% of the total hole area is alternatively covered and uncovered by the tooth while the remaining $45 \%$ are permanently uncovered. For an easier understanding, Fig. 2 shows the relative positions of the toothed wheel and the hole for different CSR. Because the modulations created by the siren travel through the plenum and the swirlers before entering the chamber, it is difficult to provide a precise relationship between the CSR and the modulation amplitude inside the chamber. Using PDA measurements [42] at a reference point in the swirling jet in the chamber ( $X=10 \mathrm{~mm}, \mathrm{Z}=-20 \mathrm{~mm}$ ), CSR $88 \%$ (the maximum) can nevertheless be associated with axial velocity fluctuations of about $12 \%$ of the mean axial velocity value. Further information regarding the characterization of the siren-induced modulations is given in appendix A.

In this study, the burner is operated around a reference point with an air flow rate of $32 \mathrm{~g} . \mathrm{s}^{-1}$ and air is preheated so that its temperature is $473 \mathrm{~K}$ in the plenum. The fuel flow rate is kept constant at $1.02 \mathrm{~g} . \mathrm{s}^{-1}$ and no siren modulations are performed. In non-reacting conditions, the spray generated by the pressurized nozzle in the chamber for this fuel flow rate shows a Sauter Mean Diameter (SMD) of $28 \mu \mathrm{m}$ for a profile taken at $\mathrm{X}=10 \mathrm{~mm}$ [42]. This operating point corresponds to a burner power of $45 \mathrm{~kW}$ and a global equivalence ratio of 0.5 .

\section{Diagnostics}

In the present study, focus is put on the liquid fuel spray and diagnostics depending on Mie scattering are thus used with two different setups shown in Fig. 3.

The first configuration (shown on the top of Fig. 3) consists in recording the Mie scattering signal from the droplets as they cross a vertical $1 \mathrm{~mm}$-thick laser sheet in the chamber. The laser sheet is created using Nd:YAG lasers (Quantronix Condor) and the signal is recorded using a Photron Fastcam SA-5 high-speed camera with a 532( \pm 10$) \mathrm{nm}$ filter. The camera is equipped with a $50 \mathrm{~mm}, \mathrm{f}: 1.4 \mathrm{lens}$ (Nikon) and records at a frame rate of 20,000 fps. The sheet is created using two $10 \mathrm{kHz}$ lasers, the second one being shot $25 \mu \mathrm{s}$ after the first one. This setup is chosen for subsequent PIV post-processing but in the present study the results are treated as two separate $10 \mathrm{kHz}$ measurements. The 15,000 recorded images for each case have a size of $704 \times 520$ pixels where 4 pixels represent $1 \mathrm{~mm}$ in the physical space.

In order to observe the behavior of the spray injection upstream of the combustion chamber, another Mie scattering recording configuration is proposed and shown at the bottom of Fig. 3. The beam of a Nd:YAG laser with a $10 \mathrm{kHz}$ repetition rate is expanded with a diverging lens $(\mathrm{f}=-75 \mathrm{~mm})$ and sent into the diverging exit of the injector. The light scattered by the liquid fuel as it is injected is recorded by a high-speed camera (Photron Fastcam SA-5) equipped with a $105 \mathrm{~mm}$, f:5.6 lens (Nikon) and a 532( \pm 10$) \mathrm{nm}$ filter. Because of the volume lighting, quantitative spatial information cannot be extracted from such images but the high speed allows for the temporal resolution of key phenomena as will be shown in the following. 

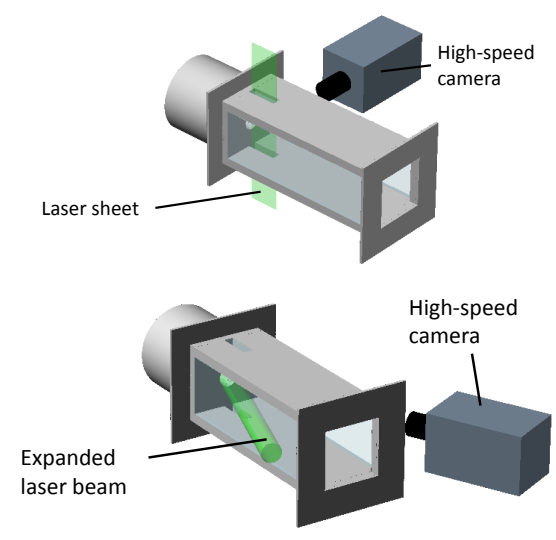

FIGURE 3. Description of the two geometrical configurations respectively dedicated to the chamber Mie scattering measurements (top) and internal Mie scattering recordings (bottom).

The flame $\mathrm{CH}^{*}$ chemiluminescence is recorded with a high speed camera (Photron Fastcam SA-X) equipped with a light intensifier (Hamamatsu C10880-03C), a $50 \mathrm{~mm}$ f:1.4 lens and a $431( \pm 10) \mathrm{nm}$ filter. The $512 \times 512$ pixels images enable the recording of a $153 \times 153$ $\mathrm{mm}$ region in the beginning of the chamber, corresponding to the same region of interest as for the chamber Mie scattering measurements. While this setup can be used to study the flame dynamics [42], only average flame images are sought in the framework of this study.

Simultaneously to the previously described spray observation techniques, the flame is also monitored using a photomultiplier (Hamamatsu, H5784-04) equipped with a $431 \pm 10 \mathrm{~nm}$ filter in order to record $\mathrm{CH}^{*}$ chemiluminescence. The pressure fluctuations in the combustion chamber are also recorded with a B\&K microphone (4938) mounted inside a semi-infinite water-cooled waveguide located at half the length of the chamber. The signals from these sensors are acquired with a NI acquisition card (PCI-MIO-16E-4) at a 16384 Hz frequency.

\section{DMD post-processing}

Dynamic Mode Decomposition (DMD) is a fairly recent linear analysis technique in the field of fluid mechanics. It consists in extracting modes from a set of time-resolved data $[43,44]$. These modes are spatial features that oscillate at a fixed frequency. It is thus a very useful tool to analyse coherent structures from a flow visualization and is therefore extensively used in the present study.

The idea behind this technique is to study the eigenvalues and eigenvectors of a linear operator $A$ that links the data at one instant with the data one time-step after. Assuming a recording of $\mathrm{N}$ snapshots, one can write the relationship between $V_{1}^{N-1}$ (the matrix regrouping the first to the penultimate snapshot) and $V_{2}^{N}$ (the matrix from the second to the last snapshot) as:

$$
V_{2}^{N}=A V_{1}^{N-1}
$$

It is however impossible to obtain $A$ only from experimental data since it would require to linearise the underlying equations. Several algorithms aim at obtaining an approximation of the eigenvalues and eigenvectors of $A$ from the data [45]. In the present study, the algorithm relies on a Singular Value Decomposition (SVD) of $V_{1}^{N-1}$. The purpose is to obtain a matrix $F$ which represents the projection of $A$ on the left singular vectors (also called the POD modes) of $V_{1}^{N-1}$. If $U \Sigma V^{*}$ (with ${ }^{*}$ denoting the Hermitian transpose operation) is the "economy-size" SVD of $V_{1}^{N-1}, F$ is defined as $F=U^{*} A U$. Plugging this definition and the SVD of $V_{1}^{N-1}$ into eq. 1, one can obtain the following expression for $F$ :

$$
F=U^{*} V_{2}^{N} V \Sigma^{-1}
$$

The eigendecomposition of $F$ can then be performed to obtain the DMD modes from the eigenvectors and their respective frequencies from the associated eigenvalues. 
Each computed DMD mode $M$ depends on both space and time coordinates and can be written as:

$$
M(x, t)=K(x) e^{i \phi(x)} e^{(a+i \omega) t}
$$

where $K(x)$ represents the oscillation amplitude which depends on the location in space, $\phi(x)$ is a quantity that represents the phase value of each point in space and $e^{(a+i \omega) t}$ is the temporal evolution of the mode. Because each mode corresponds to only one frequency $\left(\frac{\omega}{2 \pi}\right)$, it is interesting to plot a spectrum (mode norm $v s$ frequency) as an analogy with Fourier transform to obtain a quick overview of the strength of each mode. For this purpose, it is necessary to reduce the spatial and temporal dependence of each mode to only one number representing its norm. This number will then be plotted versus the mode frequency. Defining such a number is made easier by the possibility to separate the spatial evolution $\left(K(x) e^{i \phi(x)}\right)$ from the temporal evolution $\left(e^{(a+i \omega) t}\right)$. For the DMD spectra used in the present study, the mode norm is chosen as the multiplication of the $\mathrm{L}^{2}$-norm of the spatial part and the modulus of the temporal part taken at half the duration of the recording $\left(\left|e^{(a+i \omega) \frac{t_{\text {max }}}{2}}\right|=e^{a^{\frac{t_{\text {max }}}{2}}}\right)$. The $\mathrm{L}^{2}$-norm is close to an "energy" content of the mode while the choice for the temporal part enables to get rid of the non-physical fast decaying modes that can have a large spatial $\mathrm{L}^{2}$-norm but for a very limited amount of time. All the modes that will be studied in details in the present paper are stationary, meaning that for them the value of $a$ in $e^{(a+i \omega) t}$ is close to 0 .

\section{BURNER DYNAMICS}

\section{Flame and spray dynamics in the chamber}

After ignition, the flame takes a V shape (as shown in Fig. 1) and is located inside the hollow cone created by the fuel spray. The reaction zone extends upstream inside the injector. To monitor the flame dynamics, the signal from the photomultiplier is used and its power spectral density estimate is computed using Welch's method (32 Hamming windows of $0.125 \mathrm{~s}$ duration with $50 \%$ overlap). The resulting spectrum is shown on the top of Fig. 4 . It is normalized with respect to the DC component $(0 \mathrm{~Hz})$ of the signal.
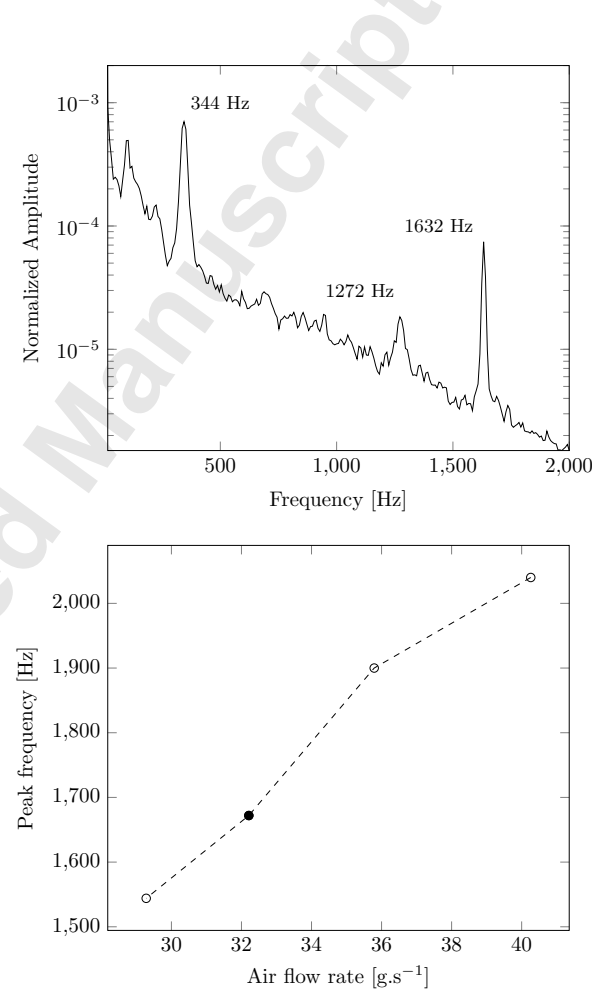

FIGURE 4. Normalized PSD (spectral resolution: $8 \mathrm{~Hz}$ ) of the photomultiplier signal (top) and frequency of the high frequency peak versus air flow rate through the burner (bottom). The filled circle represents the reference operating point. 
On the normalized PSD spectrum of the PM signal, three important peaks can be observed. Starting from the low frequencies, the first one, at $344 \mathrm{~Hz}$, is a longitudinal thermo-acoustic instability stemming from the coupling between the flame and the quarter wave mode of the combustion chamber [46]. While this phenomenon remains relatively weak for the present conditions, it can become much stronger for other operating conditions.

The second marked peak around $1272 \mathrm{~Hz}$ can only be understood if one looks at the frequency of the third one, which is $1632 \mathrm{~Hz}$. Indeed, $1272 \mathrm{~Hz}$ is close to the difference between the frequencies of the first and third peaks and can thus be linked to the interaction between the thermo-acoustic instability and the phenomenon generating the $1632 \mathrm{~Hz}$ peak. The frequency of this latter peak cannot be related to any of the burner acoustic modes and it changes depending on the air flow rate through the burner as shown by the graph at the bottom of Fig. 4. This graph is obtained by changing the air flow rate in the burner while keeping the fuel flow rate (and thus the burner power) constant, meaning that the global equivalence ratio also changes. For each point, the PSD estimate of the PM signal is computed using the same method as above and the frequency of the highest peak of the spectrum between $1200 \mathrm{and} 2200 \mathrm{~Hz}$ is plotted. The highest probed air flow rate is restricted by the lean blow-off limit. For the low values of the air flow rate (higher equivalence ratio), it becomes more and more difficult to detect a clear peak in the PM signal. As the air flow is varied, it must be noted that the amplitude of the thermo-acoustic peak also changes and that the interaction peak only appears when both the first and third peak are strong. The graph shows that the frequency of the third peak seems to increase linearly with the air flow rate in the studied range. Meanwhile, since the equivalence ratio decreases with the air flow rate, the gas temperature also decreases and so does the speed of sound. This clearly rules out any acoustic origin for the high frequency peak. Similarly, since the fuel flow rate is kept constant, it is clear that the peak does not come from the fuel spray generation system.

Since the origin of this high frequency peak seems of aerodynamical nature, similar fluctuations in the signal from the fuel droplets in the chamber may occur. A DMD (500 samples, $4 \times 4$ pixels bins) of the chamber Mie scattering recording is thus performed. The DMD spectrum from this processing can be seen in the center of Fig. 5. A tall peak can clearly be identified. This indicates that one mode has

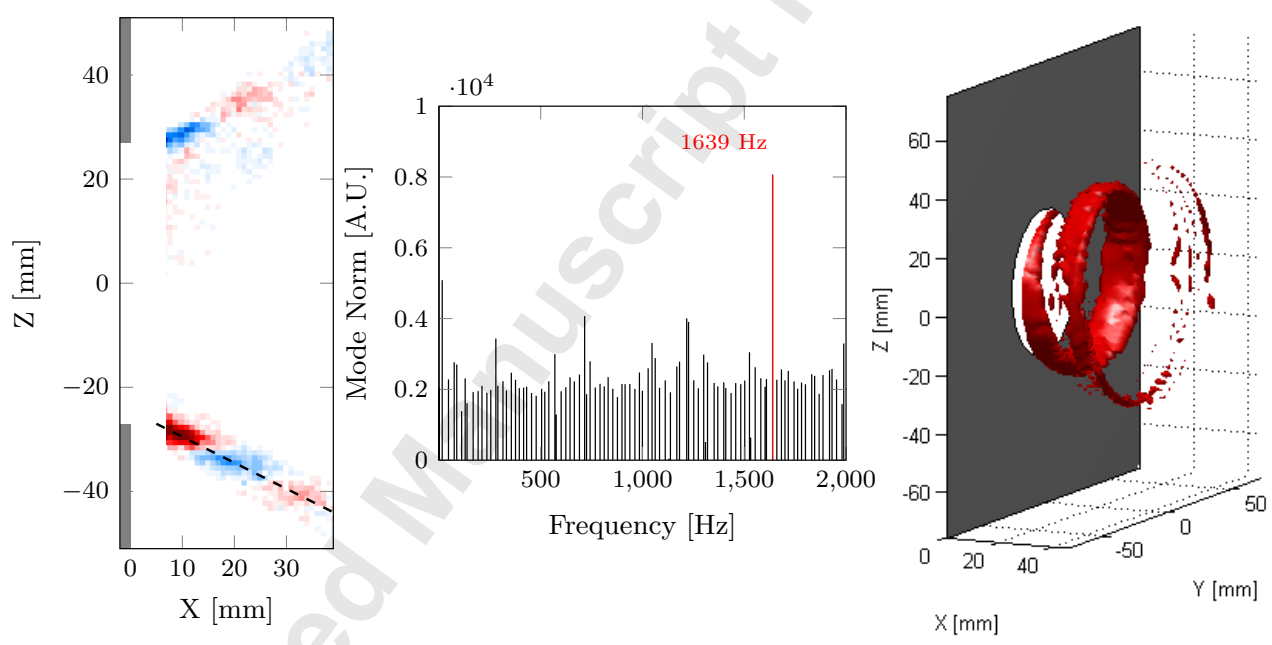

FIGURE 5. Left: Shape of the DMD mode around $1639 \mathrm{~Hz}$ for the chamber Mie scattering recording. Red (resp. blue) corresponds to positive (resp. negative) fluctuations around the mean value. The black dashed line is used in the last section for a local analysis. Center: DMD spectrum of the chamber Mie scattering recording. Right: 3D reconstruction of the $1639 \mathrm{~Hz}$ mode based on a direct mapping between the phase angle of the DMD mode and the physical angle in the Y-Z plane. The isosurface shown in red corresponds to positive fluctuations above a threshold set at 5\% of the maximum value.

a higher norm than the others and consequently that the motions it represents tend to be dominant in the Mie scattering recording. To analyze these motions, the mode shape $\left(\operatorname{Re}\left(K(x) e^{i \phi(x)}\right)\right)$ is shown on the left of Fig. 5.

The mode shows alternating and antisymmetric red and blue patches corresponding to Mie scattering fluctuations above average and below average respectively. These structures are located in two arms, corrresponding to a cut of the hollow cone shape of the spray. As the phase angle evolves due to the effect of the temporal part $e^{i \omega t}$, the structures move downstream along each arm. These 
particular mode shape and behavior can be associated with the cut of a helical structure rotating around the chamber axis. The axial distance between two consecutive red (or blue) patches then corresponds to the pitch of the helix, which in the present case is around $20 \mathrm{~mm}$. Given the structure rotational frequency of $1639 \mathrm{~Hz}$, the corresponding axial velocity is $33 \mathrm{~m} . \mathrm{s}^{-1}$. Interestingly, this value also corresponds to the average spray axial velocity for the present operating conditions [42]. The displacement of the patches along each arm can thus be associated to a physical motion: the progress of the fuel droplets inside the chamber.

To obtain a better view of the structure, a 3D reconstruction is performed following a procedure introduced in Moeck et al. [47]. It is assumed that what is observed in the monitored plane for different phase angles is similar to what is happening in differently inclined planes at a given phase angle. This assumption is made possible by the rotation of the structure around the chamber axis. It is then possible to associate each phase angle of the DMD mode with a physical angle in the Y-Z plane. The right side of Fig. 5 shows an isosurface of positive fluctuations above $5 \%$ of the maximum.

The 3D reconstruction clearly shows the helical shape of the structure in the fuel spray inside the combustion chamber. Its effect seems to quickly decay after $40 \mathrm{~mm}$. This distance also roughly corresponds to the decay of the global Mie scattering signal because of liquid fuel vaporization and mixing. Further analysis of this helical structure's decay is carried out in the following sections.

Because the flow is swirling, such a helical structure is likely to be associated with a Precessing Vortex Core. However, none of the velocity measurements inside the chamber show gaseous flow velocity fluctuations that would be compatible with such a structure [42]. This is detailed in Appendix B. Furthermore, a Strouhal number can be computed based on the structure frequency, the injector outlet diameter $(50 \mathrm{~mm})$ and a bulk velocity $\left(14 \mathrm{~m} \cdot \mathrm{s}^{-1}\right.$, using the injector outlet surface and the air flow rate). Its value is around 6 which is much higher than the typical results [16]. This indicates that the frequency of the structure is too high to have its origin in a PVC created by a vortex breakdown due to the sudden expansion in the combustion chamber. The source of the structure must thus come from inside the injector where smaller sections and higher velocities are more compatible with this higher frequency.

\section{Upstream spray dynamics}

The internal Mie scattering setup is used to observe the spray behavior inside the injector, near the exit of the pilot nozzle. The associated field of view is shown in Fig. 6 a) where the limit of the pilot stage is highlighted with a red circle. Inside the right half of this circle, it is possible to observe the tip of the pilot fuel nozzle and a relatively strong signal coming from the fuel that has just been injected.

Dynamic Mode Decomposition (500 samples, $2 \times 2$ pixels bins) is applied to these internal Mie scattering images in order to highlight the dynamical behavior of the early spray. In the DMD spectrum, a peak at $1645 \mathrm{~Hz}$ stands out. The associated mode, shown in Fig. 6 b), thus corresponds to an upstream part of the structure observed in Fig. 5. The black circle is the limit of the pilot stage, as shown in red in Fig. 6 a). The mode shows a yin-and-yang-like structure inside the pilot stage, rotating in the clockwise direction. The center of rotation seems to be located at the tip of the pilot fuel nozzle, that is slightly to the right-hand side because of the tilt of the camera. These rotating fluctuations can be seen as the result of the disturbance of the fuel injection by a precessing structure, most likely a PVC, attached to the pilot fuel nozzle and rotating in the same direction as the swirling flow. Indeed, the Strouhal number based on the parameters of the pilot stage is around 0.8 which is much more compatible with the literature results. This also explains why no gaseous velocity fluctuations are detected inside the chamber since the axial propagation of the PVC is generally limited (less than 1.5 diameters [16], which corresponds to about $25 \mathrm{~mm}$ here while the outlet of the pilot stage is around $30 \mathrm{~mm}$ before the chamber). It is to be noted that large eddy simulations simulations [48] carried out on the same experimental setup (albeit for a different operating point) also show a rotating low pressure helical region close to the pilot nozzle.

The Mie scattering fluctuations inside the injector can be used to monitor the PVC behavior as early as possible, especially its frequency. For this purpose, a $30 \times 30$ pixels averaging window, shown in red in the first frame of Fig. 6 b), is chosen inside the circle representing the limit of the pilot stage to obtain a temporal signal at a rate of $10 \mathrm{kHz}$. The rotating structure creates fluctuations in this signal each time it crosses the averaging window. The signal is then processed by a continuous wavelet transform [49] using a complex Morlet wavelet (center frequency=6; bandwidth parameter=10) as the mother. The scales are chosen to map the $1540-1740 \mathrm{~Hz}$ range with $2 \mathrm{~Hz}$ steps. A time-frequency representation of the signal is thus obtained and, for each time step, the PVC frequency is defined as the frequency associated with the highest scalogram coefficient. A histogram of the 10918 detected PVC frequency values is drawn in Fig. 6 c). The frequencies for the PVC are closely packed around $1646 \mathrm{~Hz}$ with a full width at half maximum (FWHM) of less than $12 \mathrm{~Hz}$. This is the trace of a very stable structure, explaining why the fluctuations created in the early spray and convected in the chamber keep enough coherence to be easily observable downstream.

The results shown in this section help to understand how the PVC acts in the studied combustor. By rotating near the exit of the pilot fuel nozzle, the PVC disturbs the fuel injection which then behaves as if the source of droplets was precessing instead of being fixed. 

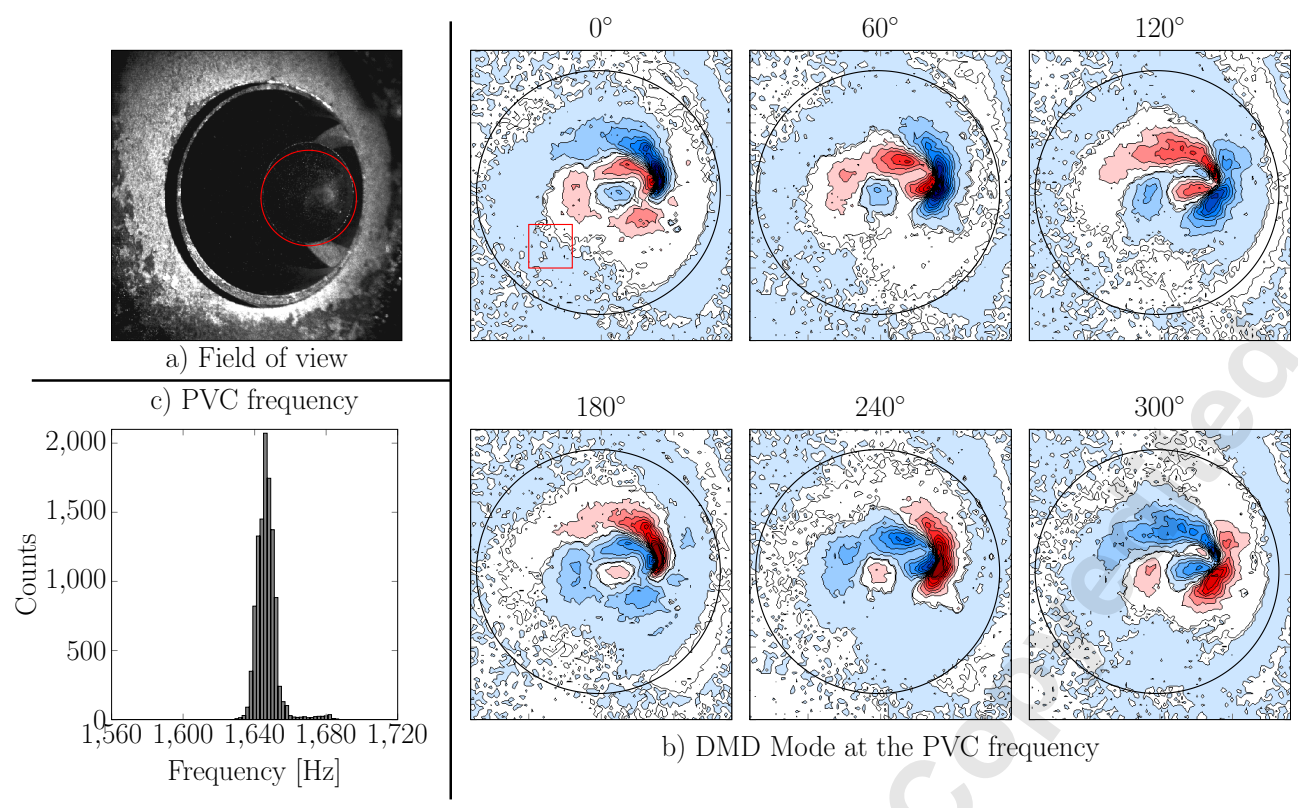

b) DMD Mode at the PVC frequency

FIGURE 6. a) Field of view of the internal Mie scattering recordings. The red circle represents the limit of the pilot stage. b) DMD mode at the PVC frequency for different phase angles from the internal Mie scattering recordings. Red (resp. blue) corresponds to positive (resp. negative) fluctuations around the mean value. The black circle is at the same position as the red one in the previous image. The red square in the first frame represents the averaging window used to obtain a temporal signal. c) Histogram of the detected PVC frequency based on a wavelet processing of the signal extracted from an averaging window in the internal Mie scattering images.

These perturbations are convected as the droplets move to the combustion chamber. This gives rise to a clear helical structure which disappears because of fuel vaporisation and mixing. It must be stressed here that this helical structure is not the PVC itself (which is damped much earlier) but its footprint that remains in the spray. The flame responds to the generated non-uniform fuel distribution which gives rise to chemiluminescence fluctuations that are detected by the photomultiplier. Since the PVC is dependent on the air flow rate, monitoring its behavior when submitted to air flow rate modulations can bring insight to the mechanisms piloting its impact on the fuel spray and then on the flame.

\section{RESPONSE TO EXTERNAL PERTURBATIONS}

In this section, the siren is used to create air flow rate modulations at a fixed frequency of $290 \pm 2 \mathrm{~Hz}$. This value is chosen to be in the same frequency range as the thermo-acoustic instability highlighted in Fig. 4 while keeping enough distance with it to avoid artificially reinforcing it and to be able to separate the two phenomena. Five different amplitude values are used: CSR 37\%, CSR 55\%, CSR $67 \%$, CSR $77 \%$ and CSR $88 \%$.

It is first necessary to determine whether the siren induced modulations strongly impact the flame and the spray from an average point of view. Indeed, intense air flow rate fluctuations can change the way the flame is stabilized [38,50]. To analyze this potential effect, average representations of the spray and the flame without siren modulations and with full amplitude modulations are shown in Fig. 7.

The flame shape is shown as a mean $\mathrm{CH}^{*}$ chemiluminescence image. The color scale is the same for all cases and is chosen to span the interval between the overall minimum and maximum. Since the goal is to compare the flames for different CSR value, no Abel inversion is performed as it would introduce noise along the centerline and not provide useful additional information for the comparison. The images thus correspond to the signal integrated over the line of sight. For this reason, they are similar to the color photography shown in Fig. 1. The V shape of the flame is clearly visible and remains the same regardless of the siren modulation amplitude. The air flow rate fluctuations have thus no impact on the average flame behavior.

To show the spray shape in green, a RMS image of each Mie scattering recording is first computed. This was shown to be more representative of the spray than an average image since the signal is generated by relatively sparse droplets. A threshold is then computed, 


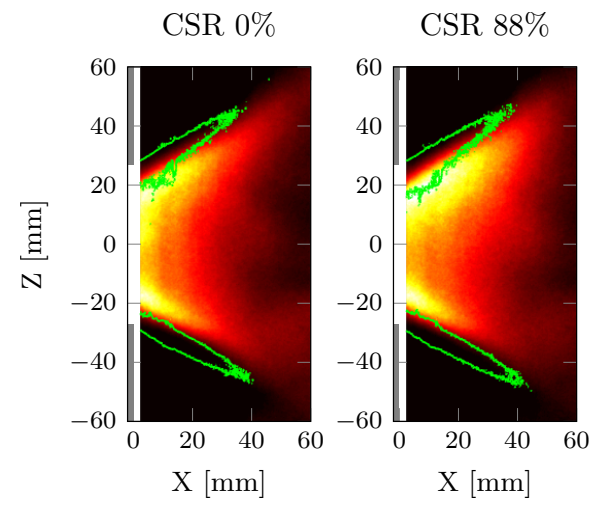

FIGURE 7. Average $\mathrm{CH}^{*}$ chemiluminescence images (shades of red and yellow) and spray contour based on a RMS image of Mie scattering for different amplitudes of the siren-induced fluctuations.

corresponding to $15 \%$ of the maximum RMS value for CSR 0\%, and applied to both images to yield the green contours in Fig. 7. The first observation that can be made is the fact that the flame is placed inside the cone of droplets. The inner part of the spray is thus interacting with the flame while the outer part is in contact with burnt gases from the outer recirculation zones. This position, as well as the penetration length of the spray, is independent of the siren-induced modulations. The only effect of the modulations one might observe could be a slight increase in spray thickness but it remains barely discernible. It is thus shown that the air flow rate modulations generated by the siren do not strongly impact the flame and spray stabilization processes and that comparisons between results for different CSR values can then be easily drawn.

\section{Global dynamics of the burner}

Using the photomultiplier signal recorded during the six studied runs (CSR 0\%, CSR 37\%, CSR 55\%, CSR 67\%, CSR 77\% and CSR 88\%), Power Spectral Density spectra are computed using the same parameters as for Fig. 4 and shown in Fig. 8.
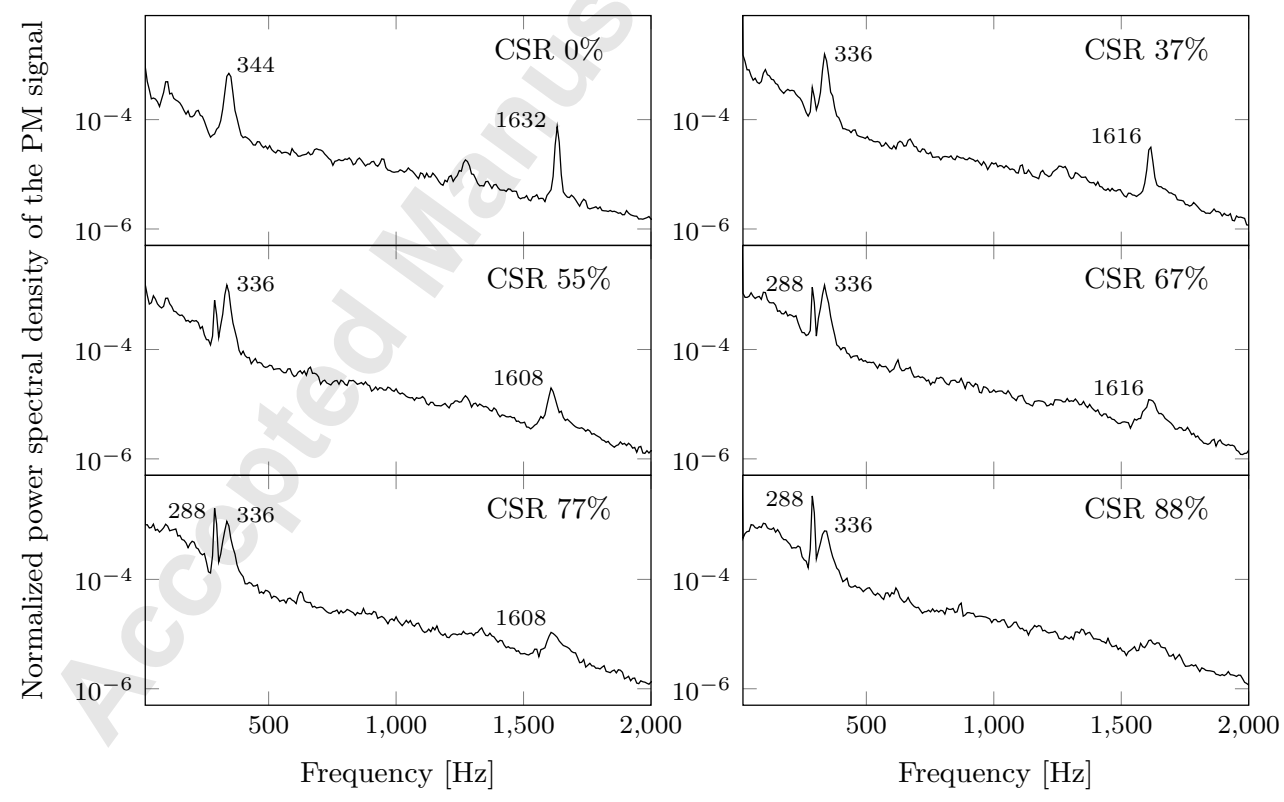

FIGURE 8. Power Spectral Density spectra (spectral resolution: $8 \mathrm{~Hz}$ ) of the photomultiplier signal for different CSR values. The spectra are normalized by their values at $0 \mathrm{~Hz}$. 
First, low-frequency content can be identified in the spectra. For CSR 0\%, the low-frequency peak at $344 \mathrm{~Hz}$ corresponds to the previously identified thermo-acoustic instability. As the modulation amplitude increases, a second low-frequency peak appears at $288 \mathrm{~Hz}$ and becomes dominant. It is the effect of the siren-induced fluctuations at $290 \mathrm{~Hz}$.

The peak associated with the PVC mode clearly stands out around $1620 \mathrm{~Hz}$ when the CSR remains low. However, as the sireninduced fluctuations increase, the peak amplitude is reduced and its width seems to increase. This phenomenon goes on until it becomes impossible to separate the peak from noise in the case of the highest amplitude modulations.

The trace of the PVC on the flame is thus strongly impacted by the siren-induced modulations which is coherent with literature results [33-36]. This however is not enough to conclude that, in this particular setup, the siren acts directly on the PVC. Indeed, the fuel spray acts as a proxy between the PVC and the flame and such a result can also stem from a change in the spray vaporization or mixing behavior due to the siren. In order to monitor potential changes in the fuel spray, a more local analysis is performed.

\section{Local evolution of the spray}

For each of the CSR values, chamber Mie scattering recordings are performed and post-processed using DMD (500 samples, 44 pixels bins). Since each recording contains a total of 15000 images, it is possible to perform 30 separate DMD for each CSR value.

To follow the local evolution of the PVC trace on the fuel spray, the resulting oscillations along one arm of the spray are analyzed. For this purpose, a line starting at $(X=5 \mathrm{~mm} ; Z=-25 \mathrm{~mm})$ and ending at $(X=45 \mathrm{~mm} ; Z=-45 \mathrm{~mm})$ is defined. It follows the direction of the bottom arm of the fuel spray and is shown in Fig. 5. Every $2 \mathrm{~mm}$ in the axial direction, $2 \times 2 \mathrm{~mm}$ interrogation windows are placed along this line. In each of these windows, the amplitude of the DMD mode $(K(x)$ in equation 3$)$ at the PVC frequency is computed and averaged. This enables to quantify the decay of the fuel spray fluctuations created by the PVC as they progress along the spray and thus to indirectly monitor the mixing and vaporization processes.

Since several DMD are performed for each CSR value, their results are averaged and plotted on the top graph of Fig. 9. Also, 95\% confidence intervals are computed and some of them are shown to give an overview of the precision of the results.

The graph clearly shows a decay of the Mie scattering intensity oscillations at the PVC frequency as they progress inside the chamber. They have almost disappeared after less than $40 \mathrm{~mm}$ in the axial direction as already observed in Fig. 5.

Apart from the CSR 37\% condition which seems to show higher oscillation amplitudes (note that this is inconclusive given the size of the confidence interval), the curves are sorted by their CSR order. This means that the siren-generated modulations amplitude is correlated with a decrease of the PVC trace on the spray. This in turn explains why the flame also shows a lower response to the PVC when high amplitude external perturbations are present.

To analyze more precisely the decay of the PVC trace on the spray, it is interesting to normalize the previous curves by their value at $\mathrm{X}=5 \mathrm{~mm}$, as shown at the bottom of Fig. 9. This allows to observe the relative damping of the oscillations as they progress inside the chamber.

Despite the differences in the siren-induced modulations amplitude, all the points collapse onto the same curve. This indicates that the shapes of the corresponding modes do not seem strongly impacted. Applying the same post-processing to the average Mie scattering images rather than to the DMD modes gives the green curve which is interestingly undistinguishable from the others. The trend all the curves follow is made of two slopes: first a steep decay down to $20 \%$ of the first value at $\mathrm{X}=20 \mathrm{~mm}$ and a flatter part afterwards. This two-step decay might be explained by the fact that the flame is strongly anchored inside the injector. Average $\mathrm{CH}^{*}$ chemiluminescence images indeed seem to show that the heat release is stronger between $X=0 \mathrm{~mm}$ and $X=20 \mathrm{~mm}$ than between $X=20 \mathrm{~mm}$ and $X=40 \mathrm{~mm}$ (Fig. 7). The vaporization of the droplets would thus be stronger in the first part of the chamber, explaining the two different slopes of the curve. This hypothesis nevertheless requires further investigation to be confirmed.

The fact that the relative curves corresponding to the PVC induced spray fluctuations all follow the same trend as the average Mie scattering intensity regardless of the CSR shows that the mixing and vaporization processes, responsible for the decay of the Mie scattering signal in the chamber, are little impacted by the siren induced modulations. Changes in the mixing and vaporization behavior thus cannot be responsible for the decrease of the PVC trace on the spray with the modulation amplitude. The reason for this decrease must be found upstream of the chamber.

\section{Upstream spray behavior}

Figure 10 shows the effect of the siren induced modulations on the dominant frequencies in the internal Mie scattering signal based on the averaging window defined in section and shown in the first frame of Fig. $6 \mathrm{~b}$ ). The mother wavelet chosen for the analysis is 

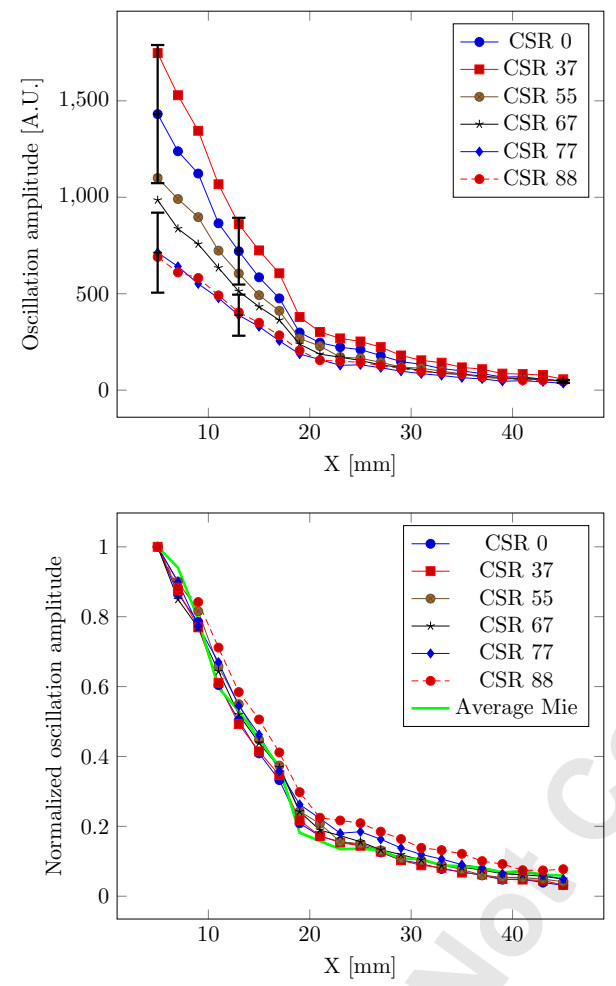

FIGURE 9. Top: mean Mie scattering oscillation amplitude of the PVC trace DMD modes in the bottom arm of the spray versus axial position when the siren amplitude is changed. The error bars represent $95 \%$ confidence intervals. Bottom: curves from the previous plot normalized by their respective values at $\mathrm{X}=5 \mathrm{~mm}$. The green line is the result of the same processing applied to the average Mie scattering images.

the same complex Morlet one (center frequency=6; bandwidth parameter=10) and the scales are now chosen to span the $1250-2050 \mathrm{~Hz}$ interval with $2 \mathrm{~Hz}$ steps.

For CSR 0\%, the dominant frequency distribution corresponds to what was already observed in Fig. 6, as expected. As the sireninduced modulations increase however, the peak tends to becomes smaller and the distribution widens. The median of the distribution remains quite stable and its small variations can be attributed to minor temperature differences between runs. The frequency spread is on the contrary already very visible for CSR $37 \%$ with an interdecile range (IDR) of $56 \mathrm{~Hz}$ while it was only $12 \mathrm{~Hz}$ for CSR $0 \%$. The IDR then steadily increases to reach $110 \mathrm{~Hz}$ for CSR $77 \%$ and even $332 \mathrm{~Hz}$ for CSR $88 \%$. For the latter case, however, the histogram shows detected frequencies all along the probed frequency range (and especially below $1400 \mathrm{~Hz}$ ). This indicates that the signal coming from the PVC is barely detectable and competes with the background noise or other weaker fluctuations. Siren-induced modulations have thus an impact on the PVC itself and tend to destroy it before it can create spray fluctuations. As shown by the histograms, this destruction occurs through a spread in frequency which prevents the structure from retaining coherence over several cycles and imparting steady fluctuations on the fuel droplets.

\section{CONCLUSION}

With the help of high-speed Mie scattering recordings and Dynamic Mode Decomposition as the main post-processing tool, the impact of the PVC on the fuel spray in a gas turbine model combustor is studied. The structure is first characterized and its response to artificially generated air flow rate modulations is studied.

First, it is shown that the PVC takes roots onto the fuel nozzle, inside the injector. From a gas flow velocity point of view, its effect is not detected in the chamber although the flame shows heat release fluctuations at its frequency. The missing link lies in the fuel spray that is disturbed by the PVC at the moment of its injection. Because fuel droplets have inertia and thus require time to reach equilibrium and uniform mixing with the gas flow, they can carry the fluctuations created by the PVC further in the chamber, up to $40 \mathrm{~mm}$. This 
CSR 0\%

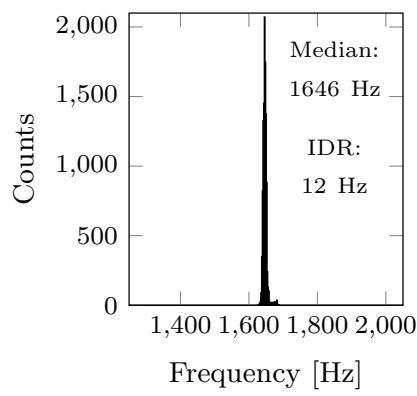

CSR $67 \%$

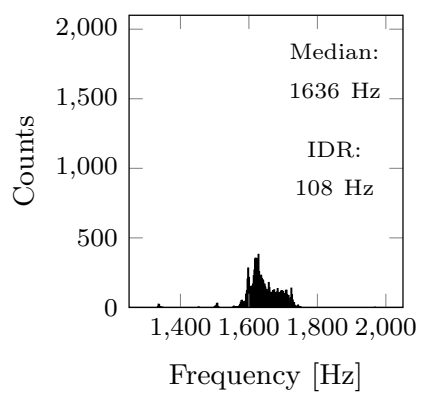

CSR $37 \%$

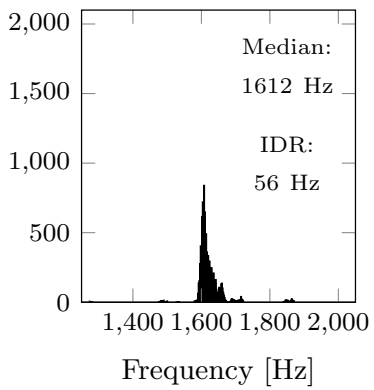

CSR $77 \%$

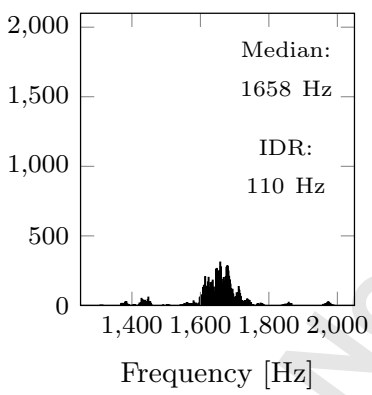

CSR 55\%

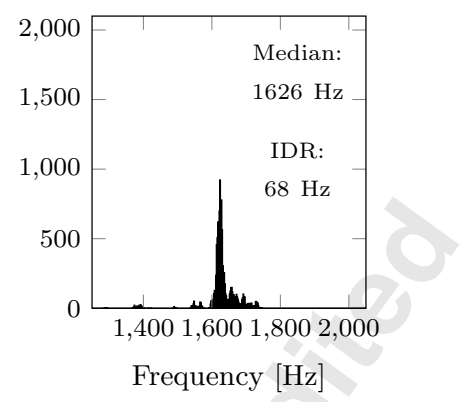

CSR $88 \%$

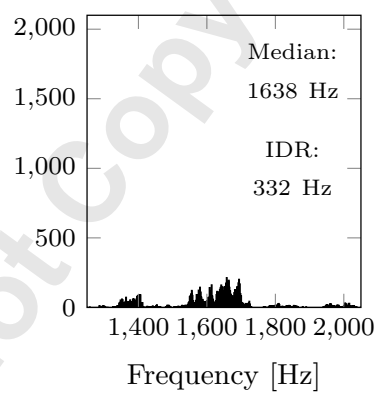

FIGURE 10. Histograms of the detected PVC frequency based on a wavelet processing of the signal extracted from an averaging window in the internal Mie scattering images. Median and interdecile range (IDR) values are given for a quantitative assessment of the location and scale of the distribution.

non-uniform fuel feeding is responsible for heat release intensity fluctuations.

The fact that the spray acts as a transportation mechanism to carry the fluctuations generated by the PVC to the flame can explain why the flame shows a response to the PVC even though it is in an attached state. Indeed, the density fluctuations created by the flame (which are considered to be a damping mechanism for the PVC) are located downstream of the root of the PVC (the fuel injector). The aerodynamical structure itself and the flame thus seldom interact and the coupling between them happens only in one direction through the fuel spray. This emphasizes the fact that one needs to exert great care before applying results observed with gaseous reactants to two-phase combustion.

To simulate a potential increase in thermo-acoustic instability amplitude, the burner is submitted to artificial air flow rate fluctuations with the help of a siren. This leads to a spread of the PVC frequency, meaning that the structure is loosing coherence. Therefore, the PVC has a weaker impact on the spray and thus on the flame. This spread in frequency can explain how acoustic forcing (and to a lesser extent thermo-acoustic instabilities) may lead to the destruction of the PVC, as observed in the literature. In the present case, it is interesting to note that the spray mixing and vaporization processes remain independent of the artificial modulations amplitude, indicating that the fuel droplets can still carry the PVC generated fluctuations from the inside of the injector to the chamber.

The post-processing methods used in this study have proven particularly useful to study the effects of the PVC in the present burner. The next step is now to apply them to study the effect of naturally-occurring thermo-acoustic instabilities of different amplitudes on the interactions between the PVC, the fuel spray and the flame. As this requires changing the equivalence ratio through the fuel flow rate or the air flow rate, modifications of the PVC structure or of the spray behavior are expected. This would enable to assess the generality of the findings from the present study, carried out with constant air and fuel flow rates. As it is also shown, the fuel spray is a key element to transport the PVC generated fluctuations to the flame. Further information on the interactions between the aerodynamical structure and the early spray need therefore to be obtained. The lack of optical access inside the injector remains however a key issue for such a 
study.

\section{ACKNOWLEDGMENT}

The authors would like to gratefully acknowledge Safran/Snecma (presently Safran Aircraft Engines) for supporting this study. We also thank Fabrice Giuliani of CBOne for designing, making and helping set up the siren device as well as Philippe Scouflaire for his help with the diagnostics hardware.

\section{REFERENCES}

[1] Correa, S. M., 1998. "Power generation and aeropropulsion gas turbines: From combustion science to combustion technology". Symposium (International) on Combustion, 27(2), Jan., pp. 1793-1807.

[2] Tacina, R. R., 1990. "Low NOx potential of gas turbine engines". AIAA Aerospace Sciences Meeting and Exhibit, 90.

[3] Lefebvre, A. H., 1995. "The Role of Fuel Preparation in Low-Emission Combustion". Journal of Engineering for Gas Turbines and Power, 117(4), Oct., pp. 617-654.

[4] Moore, M. J., 1997. "Nox emission control in gas turbines for combined cycle gas turbine plant". Proceedings of the Institution of Mechanical Engineers, Part A: Journal of Power and Energy(211), Feb., pp. 43-52.

[5] Fritz, J., Kröner, M., and Sattelmayer, T., 2004. "Flashback in a Swirl Burner With Cylindrical Premixing Zone". Journal of Engineering for Gas Turbines and Power, 126(2), p. 276.

[6] Sommerer, Y., Galley, D., Poinsot, T., Ducruix, S., Lacas, F., and Veynante, D., 2004. "Large eddy simulation and experimental study of flashback and blow-off in a lean partially premixed swirled burner". Journal of Turbulence, 5, Oct.

[7] Galley, D., Ducruix, S., Lacas, F., and Veynante, D., 2011. "Mixing and stabilization study of a partially premixed swirling flame using laser induced fluorescence". Combustion and Flame, 158(1), Jan., pp. 155-171.

[8] Candel, S., 2002. "Combustion dynamics and control: progress and challenges". Proceedings of the Combustion Institute, 29(1), pp. $1-28$.

[9] Lieuwen, T. C., and Yang, V., 2005. "Combustion instabilities in gas turbine engines(operational experience, fundamental mechanisms and modeling)". Progress in astronautics and aeronautics.

[10] Froud, D., O'doherty, T., and Syred, N., 1995. "Phase averaging of the precessing vortex core in a swirl burner under piloted and premixed combustion conditions". Combustion and Flame, 100(3), pp. 407-412.

[11] Wegner, B., Maltsev, A., Schneider, C., Sadiki, A., Dreizler, A., and Janicka, J., 2004. "Assessment of unsteady RANS in predicting swirl flow instability based on LES and experiments". International Journal of Heat and Fluid Flow, 25(3), June, pp. 528-536.

[12] Liang, H., and Maxworthy, T., 2005. "An experimental investigation of swirling jets". Journal of Fluid Mechanics, 525, Feb., pp. 115-159.

[13] Wang, S., Yang, V., Hsiao, G., Hsieh, S.-Y., and Mongia, H. C., 2007. "Large-eddy simulations of gas-turbine swirl injector flow dynamics". Journal of Fluid Mechanics, 583, July, p. 99.

[14] Valera-Medina, A., Syred, N., and Griffiths, A., 2009. "Visualisation of isothermal large coherent structures in a swirl burner". Combustion and Flame, 156(9), Sept., pp. 1723-1734.

[15] Syred, N., and Beer, J. M., 1974. "Combustion in swirling flows: a review". Combustion and Flame, 23(2), pp. 143-201.

[16] Syred, N., 2006. "A review of oscillation mechanisms and the role of the precessing vortex core (PVC) in swirl combustion systems". Progress in Energy and Combustion Science, 32(2), Jan., pp. 93-161.

[17] Syred, N., Gupta, A. K., and Beér, J. M., 1975. "Temperature and density gradient changes arising with the precessing vortex core and vortex breakdown in swirl burners". Symposium (International) on Combustion, 15(1), Jan., pp. 587-597.

[18] Selle, L., 2004. "Compressible large eddy simulation of turbulent combustion in complex geometry on unstructured meshes". Combustion and Flame, 137(4), June, pp. 489-505.

[19] Oberleithner, K., Terhaar, S., Rukes, L., and Oliver Paschereit, C., 2013. "Why Nonuniform Density Suppresses the Precessing Vortex Core". Journal of Engineering for Gas Turbines and Power, 135(12), Sept., p. 121506.

[20] Manoharan, K., Hansford, S., O' Connor, J., and Hemchandra, S., 2015. "Instability Mechanism in a Swirl Flow Combustor: Precession of Vortex Core and Influence of Density Gradient". In ASME Turbo Expo 2015: Turbine Technical Conference and Exposition, ASME, pp. V04AT04A073+.

[21] Oberleithner, K., Stöhr, M., Im, S. H., Arndt, C. M., and Steinberg, A. M., 2015. "Formation and flame-induced suppression of the precessing vortex core in a swirl combustor: Experiments and linear stability analysis". Combustion and Flame, 162(8), Aug., pp. 3100-3114. 
[22] Syred, N., Fick, W., O’Doherty, T., and Griffiths, A. J., 1997. "The Effect of the Precessing Vortex Core on Combustion in a Swirl Burner". Combustion Science and Technology, 125(1-6), May, pp. 139-157.

[23] Stöhr, M., Boxx, I., Carter, C., and Meier, W., 2011. "Dynamics of lean blowout of a swirl-stabilized flame in a gas turbine model combustor". Proceedings of the Combustion Institute, 33(2), Jan., pp. 2953-2960.

[24] Taamallah, S., Shanbhogue, S. J., and Ghoniem, A. F., 2016. "Turbulent flame stabilization modes in premixed swirl combustion: Physical mechanism and Karlovitz number-based criterion”. Combustion and Flame, 166, Apr., pp. 19-33.

[25] Stöhr, M., Boxx, I., Carter, C. D., and Meier, W., 2012. "Experimental study of vortex-flame interaction in a gas turbine model combustor". Combustion and Flame, 159(8), Aug., pp. 2636-2649.

[26] Renaud, A., Ducruix, S., Scouflaire, P., and Zimmer, L., 2015. "Flame shape transition in a swirl stabilised liquid fueled burner". Proceedings of the Combustion Institute, 35(3), pp. 3365-3372.

[27] Terhaar, S., Oberleithner, K., and Paschereit, C. O., 2015. "Key parameters governing the precessing vortex core in reacting flows: An experimental and analytical study". Proceedings of the Combustion Institute, 35(3), pp. 3347-3354.

[28] An, Q., Kwong, W. Y., Geraedts, B. D., and Steinberg, A. M., 2016. "Coupled dynamics of lift-off and precessing vortex core formation in swirl flames". Combustion and Flame, 168, June, pp. 228-239.

[29] Stöhr, M., Arndt, C. M., and Meier, W., 2013. "Effects of Damköhler number on vortexflame interaction in a gas turbine model combustor". Proceedings of the Combustion Institute, 34(2), Jan., pp. 3107-3115.

[30] Steinberg, A. M., Boxx, I., Stöhr, M., Carter, C. D., and Meier, W., 2010. "Flowflame interactions causing acoustically coupled heat release fluctuations in a thermo-acoustically unstable gas turbine model combustor". Combustion and Flame, 157(12), Dec., pp. 2250-2266.

[31] Boxx, I., Arndt, C. M., Carter, C. D., and Meier, W., 2012. "High-speed laser diagnostics for the study of flame dynamics in a lean premixed gas turbine model combustor". Experiments in Fluids, 52(3), Mar., pp. 555-567.

[32] Johchi, A., Zimmer, L., and Tanahashi, M., 2014. "Investigation on the Acoustic Behavior of a Turbulent Swirl-Stabilized Combustor Fed with Liquid Fuel". In 17th International Symposium on Applications of Laser Techniques to Fluid Mechanics, Lisbon, Portugal.

[33] Khalil, S., Hourigan, K., and Thompson, M. C., 2006. "Response of unconfined vortex breakdown to axial pulsing". Physics of Fluids, 18(3), Mar., p. 038102.

[34] Moeck, J. P., Bourgouin, J.-F., Durox, D., Schuller, T., and Candel, S., 2012. "Nonlinear interaction between a precessing vortex core and acoustic oscillations in a turbulent swirling flame". Combustion and Flame, 159(8), Aug., pp. $2650-2668$.

[35] Alekseenko, S. V., Dulin, V. M., Kozorezov, Y., and Markovich, D. M., 2012. "Effect of High-Amplitude Forcing on Turbulent Combustion Intensity and Vortex Core Precession in a Strongly Swirling Lifted Propane/Air Flame". Combustion Science and Technology, 184(10-11), Oct., pp. 1862-1890.

[36] Terhaar, S., Ćosić, B., Paschereit, C. O., and Oberleithner, K., 2016. "Suppression and excitation of the precessing vortex core by acoustic velocity fluctuations: An experimental and analytical study". Combustion and Flame, 172, Oct., pp. $234-251$.

[37] Iudiciani, P., and Duwig, C., 2011. "Large Eddy Simulation of the Sensitivity of Vortex Breakdown and Flame Stabilisation to Axial Forcing". Flow, Turbulence and Combustion, 86(3-4), Apr., pp. 639-666.

[38] Renaud, A., Ducruix, S., and Zimmer, L., 2017. "Bistable behaviour and thermo-acoustic instability triggering in a gas turbine model combustor". Proceedings of the Combustion Institute, 36(3), pp. 3899-3906.

[39] Hermeth, S., Staffelbach, G., Gicquel, L. Y. M., Anisimov, V., Cirigliano, C., and Poinsot, T., 2014. "Bistable swirled flames and influence on flame transfer functions". Combustion and Flame, 161(1), Jan., pp. 184-196.

[40] Terhaar, S., Krüger, O., and Paschereit, C. O., 2015. "Flow Field and Flame Dynamics of Swirling Methane and Hydrogen Flames at Dry and Steam Diluted Conditions". Journal of Engineering for Gas Turbines and Power, 137(4), p. 041503.

[41] Giuliani, F., Lang, A., Gradl, K. J., Siebenhofer, P., and Fritzer, J., 2012. "Air Flow Modulation for Refined Control of the Combustion Dynamics Using a Novel Actuator". Journal of Engineering for Gas Turbines and Power, 134.

[42] Renaud, A., 2015. "Étude de la stabilisation des flammes et des comportements transitoires dans un brûleur étagé à combustible liquide à l'aide de diagnostics rapides". PhD thesis, Paris Saclay.

[43] Schmid, P. J., 2010. "Dynamic mode decomposition of numerical and experimental data". Journal of Fluid Mechanics, 656, July, pp. 5-28.

[44] Schmid, P. J., 2011. "Application of the dynamic mode decomposition to experimental data". Experiments in Fluids, 50(4), Feb., pp. 1123-1130.

[45] Rowley, C. W., Mezić, I., Bagheri, S., Schlatter, P., and Henningson, D. S., 2009. "Spectral analysis of nonlinear flows". Journal of Fluid Mechanics, 641, Nov., p. 115.

[46] Providakis, T., 2013. "Compétition entre Structures Aérodynamiques et Modes Acoustiques dans une Flamme Swirlée: Influence 
de la Répartition de Carburant". PhD thesis, Ecole Centrale Paris, Châtenay Malabry, France, Apr.

[47] Moeck, J. P., Bourgouin, J.-F., Durox, D., Schuller, T., and Candel, S., 2013. "Tomographic reconstruction of heat release rate perturbations induced by helical modes in turbulent swirl flames". Experiments in Fluids, 54(4), Apr.

[48] Cheneau, B., Vié, A., and Ducruix, S., 2015. "Large Eddy Simulations of a Liquid Fuel Swirl Burner: Flame Characterization for Pilot and Multipoint Injection Strategies". In ASME Turbo Expo 2015: Turbine Technical Conference and Exposition, American Society of Mechanical Engineers, p. V04AT04A064.

[49] Grossmann, A., Kronland-Martinet, R., and Morlet, J., 1989. "Reading and understanding continuous wavelet transforms". In Wavelets. Springer, pp. 2-20.

[50] Hardalupas, Y., Selbach, A., and Whitelaw, J. H., 2000. "Liquid-fuelled flames with imposed air oscillations". In Laser techniques applied to fluid mechanics. Springer, pp. 403-416.

\section{A Siren characterization Velocity Fluctuations}

As mentioned in the Diagnostics section, the tomographic Mie scattering data can also be processed with a PIV algorithm. Because a large quantity of droplets is required for this, only non-reacting data are analyzed. The PIV fields in Fig. 11 and 12 are computed using $20 \times 20$ pixels windows with $60 \%$ overlap to yield a velocity vector every $2 \mathrm{~mm}$.

Figure 11 presents the average spray velocity field in the absence of siren-induced modulations. It must be noted here that the fuel droplets play the role of the PIV seeding and, since they have a non-negligible Stokes number, the spray velocity is not representative of the air flow velocity. This can be seen in comparison with Fig. 14.
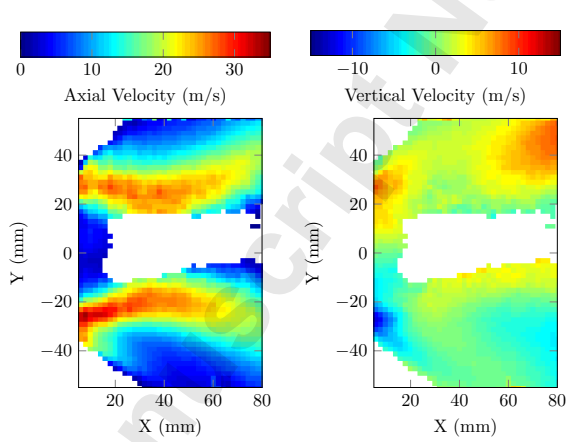

FIGURE 11. Mean axial (left) and vertical (right) spray velocities from the PIV treatment of the Mie scattering fields. A threshold has been set at $2 \%$ of vector validation explaining the presence of white zones.

First, it can be seen that no velocity information is available in the recirculation zones, as expected since no droplets are present there. In the jet region, the axial velocities are high (about $30 \mathrm{~m} / \mathrm{s}$ ), except in the external parts of the jet after $\mathrm{X}=25 \mathrm{~mm}$ where they remain around $10 \mathrm{~m} / \mathrm{s}$. The vertical velocities are globally symmetrical and, in the jet region, an area with much lower values can be highlighted which shows that the spray becomes less divergent with its axial progress.

For the recording at CSR 88\%, the axial and vertical velocity data from the PIV treatment are post processed by Dynamic Mode Decomposition on 500 samples and the components of the mode at the siren frequency can be seen in Fig. 12.

The axial velocity fluctuations have a symmetrical shape with amplitudes up to $7 \mathrm{~m} / \mathrm{s}$ which means the droplets are alternatively accelerated and slowed with the air flow rate modulations, as expected. The vertical velocity fluctuations are more interesting to analyze because they have an antisymmetric shape, meaning that the spray is alternatively contracting and expanding. From the beginning of the chamber, it can be seen that the contracting phase corresponds to low axial velocities, that is, a low air flow rate. This effect comes from the fact that the siren is placed before the swirlers. The air flow rate modulations are converted into azimuthal fluctuations which in turn generate radial velocity fluctuations by centrifugal effect. Compared to the results in Fig. 11, it appears that the modulations represent roughly $10 \%$ of the average value. 


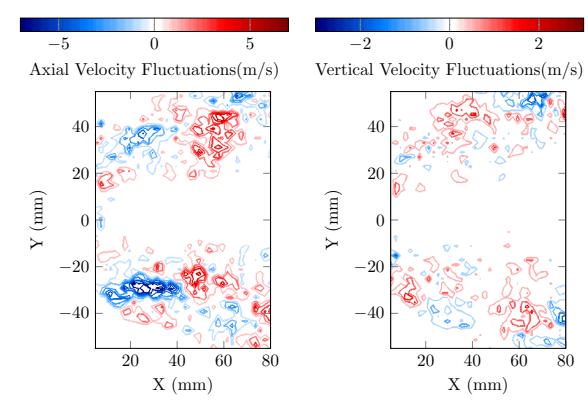

FIGURE 12. Contour plot of the DMD mode associated with the siren (CSR 88\%) for the axial (left) and vertical (right) spray velocities from the PIV treatment of the non-reacting Mie scattering fields.

\section{Acoustic fluctuations}

The combustion chamber is equipped with a microphone placed at half of its length. Using this microphone, Sound Pressure Level (SPL) values can be computed for $4 \mathrm{~s}$ long recordings for each value of the siren amplitude in non-reacting and reacting conditions. The resulting curves can be seen in Fig. 13.

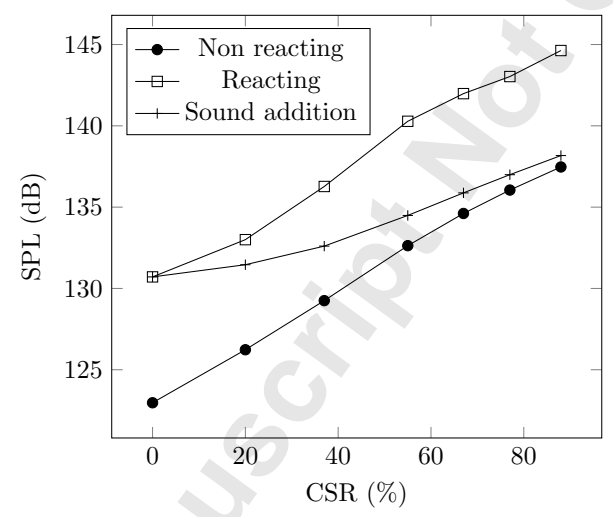

FIGURE 13. Evolution of the Sound Pressure Level in the combustion chamber as the siren-induced modulations increase.

The non-reacting curve shows, as expected, that the SPL in the chamber increases with the amplitude of the siren-induced modulations. For the maximum CSR (88\%), an increase of $14 \mathrm{~dB}$ of the SPL in the chamber can be noted. The reacting curve looks like a shift of the non-reacting one. However, because of the decibel graduation of the y axis, the implications of this shift have to be carefully studied with the help of the third curve.

On a linear scale, shifting corresponds to adding a constant value and, here, one would want to apply the same reasoning and see the reacting case curve as the addition of the non-reacting curve and a constant noise from the flame. This simple sound addition in fact corresponds to the middle curve because of the logarithmic scale. The difference between this curve and the reacting one comes from increases in the flame-generated sound, proving that the air flow rate modulations generated by the siren actually affect the flame which appears to behave like an amplifier with a roughly constant gain.

\section{B Cold flow velocity field}

For the studied air flow rate $\left(32 \mathrm{~g} . \mathrm{s}^{-1}\right)$, cold flow high-speed PIV measurements are performed. The flow is seeded with oil droplets and the recording setup is the same as the tomographic Mie scattering one presented in the Diagnostics section. Four 0.25 s-long runs are performed and then processed using a PIV algorithm from Dantec Dynamic Studio. All of the results are averaged to yield the velocity fields shown in Fig. 14. 

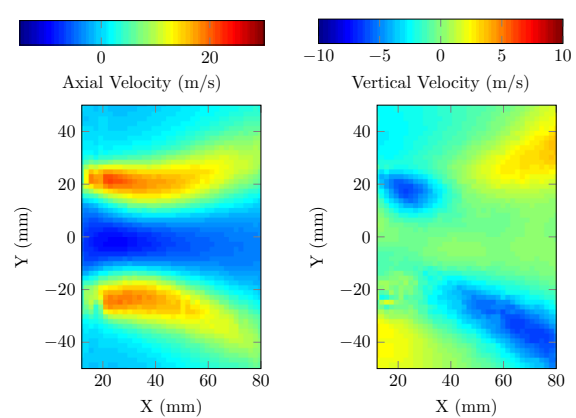

FIGURE 14. Mean axial (left) and vertical (right) cold flow velocities from PIV.

This behaviour of the cold flow velocity is very common for a confined swirling flow with a jet region characterized by high streamwise velocity values and inner and outer recirculation zones. These average fields gives an impression of the velocity field in the burner but what is interesting in the present study are the fluctuations of the velocity field, especially whether a trace of the PVC can be found in the chamber from a purely gaseous point of view.

For this purpose, the frequency at which one needs to look for the PVC must be determined. Given that the PVC is a constant Strouhal number mode, it scales with the velocity of the flow. In Fig. 6, the frequency of the PVC for a temperature of $473 \mathrm{~K}$ is found to be $1646 \mathrm{~Hz}$. Taking into account the change in air density because no preheating is used for oil-PIV, the PVC is expected to be around $1030 \mathrm{~Hz}$.

For a given position, the velocity components can be considered as time-series and thus processed with Fourier transform. One can thus obtain a spectrum of the velocity fluctuations at this position. If the velocity fluctuates because of the PVC, there will be a peak in the spectrum at the PVC frequency. By plotting the highest value in the spectra between 800 and $1200 \mathrm{~Hz}$ as a function of space, it would thus be possible to follow the trace of the PVC in the gaseous flow as it would appear as a region of almost constant frequency. This is what is shown in Fig. 15.

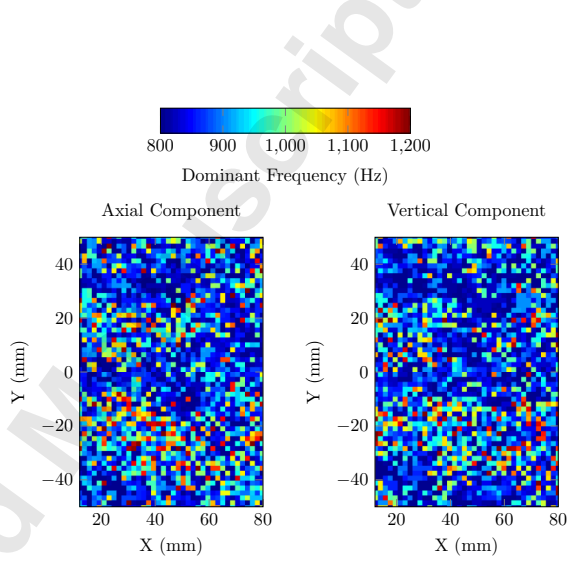

FIGURE 15. Dominant frequencies of the velocity fluctuations between 800 and $1200 \mathrm{~Hz}$ for the axial (left) and vertical (right) velocity components.

The images show mostly noise and no visible trace of the PVC that is expected to be around $1030 \mathrm{~Hz}$. Furthermore, given the spectral resolution of $10 \mathrm{~Hz}$ for this diagnostic and the fact that the PVC is expected to be very stable in frequency (see Fig. 6), it should appear in only one or two frequency bins and thus detach clearly as a region with a solid color. This is not the case here.

The cold flow PIV measurements using oil droplets as seeding thus show no evidence of a PVC in the air flow field inside the chamber. The results from Fig. 15 are confirmed by other post-processing techniques such as DMD that show no strong mode appears around $1030 \mathrm{~Hz}$. While it is impossible to prove that something does not exist, using available diagnostics and state of the art postprocessing techniques is not enough to highlight any fluctuation of the air flow field in the chamber because of the PVC. 